\begin{tabular}{|c|c|}
\hline Title & Theoretical comparison between skyrmion and skyrmionium motions for spintronics applications \\
\hline Author(s) & Y uichi, Ishida; Kenji, Kondo \\
\hline Citation & $\begin{array}{l}\text { Japanese Journal of A pplied Physics, 59(SG), SGGI04 } \\
\text { https://doi.org/10.7567/1347-4065/ab5b6b }\end{array}$ \\
\hline Issue Date & $2020-02-20$ \\
\hline Doc URL & http:/hdl.handle.net/2115/80479 \\
\hline Rights & ( 2020 The Japan Society of A pplied Physics \\
\hline Type & article (author version) \\
\hline File Information & jjap_revised_article_kondo.pdf \\
\hline
\end{tabular}

Instructions for use 


\title{
Theoretical Comparison between Skyrmion and Skyrmionium Motions for Spintronics Applications
}

\author{
Yuichi Ishida and Kenji Kondo* \\ Research Institute for Electronic Science, Hokkaido University, Kita-20, Nishi-10, Sapporo, Hokkaido, Japan
}

We discuss applications of skyrmioniums to spintronics devices in comparison with the case of skyrmions in this study. It seems that the skyrmioniums are more beneficial than the skyrmions since it is derived from the Thiele equation that the skyrmion Hall effect does not occur in the case of the skyrmioniums. However, we have found that the skyrmioniums have also the disadvantage for the spintronics devices. We cannot utilize the skyrmionium in the system with small Gilbert damping constant since micromagnetic simulation has shown that the skyrmionium structure distorts largely when the Gilbert damping constant is small. From the above reason, the skyrmioniums are not entirely more beneficial to the spintronics devices than the skyrmions. In the last of this paper, we provide a creation method of the skyrmionium by utilizing pulsed magnetic fields. We consider that this method is useful to create the skyrmioniums experimentally.

\section{Introduction}

Recently, in the field of the spintronics, magnetic skyrmions have attracted much attention and it is expected that they can be applied to spintronics devices such as logic devices and high-density memories. ${ }^{1,2)}$ This expectation originates from the following three properties of skyrmions. Firstly, skyrmions are like particles and their sizes are relatively small. Generally, the sizes of skyrmions are from $5 \mathrm{~nm}$ to $100 \mathrm{~nm} .{ }^{3)}$ Secondary, since structures of skyrmions are topologically protected, they are relatively stable against perturbations and it is difficult to deform them to other magnetic structures. Thirdly, skyrmions can be driven by lower electrical current density than domain walls. ${ }^{1)}$ The first and the second properties result in the fact that one bit of information can be represented by the existence of a skyrmion. Moreover, from the third property, it is considered that the information can be transferred by motion of skyrmions at low power consumption. Although it seems that skyrmions do not have any disadvantages, there exists a disadvantage for applying to the spintronics devices. Namely, when we move skyrmions by utilizing electrical currents, skyrmions have velocity components perpendicular to the direction of the electrical current flow depending on both Gilbert damping constant and non-adiabatic constant. This effect is well known as the skyrmion Hall effect. ${ }^{4-7)}$ In the skyrmion Hall effect, ratio of in-plane velocity components depends on material parameters. Since the skyrmion Hall effect originates from the fact that skyrmions have the topological invariant called topological charge $Q$ according to the Thiele equation, it is expected that the skyrmion Hall effect does not occur in the cases of magnetic structures which do not have the topological charge.

Magnetic skyrmioniums also have particle-like nature as the same as magnetic skyrmions and their topological charges are equal to zero. ${ }^{8-11)}$ Therefore, skyrmioniums could overcome the above disadvantage of skyrmions. Namely, it is expected that skyrmioniums motion could be always parallel with the electrical current flow. This means that they could be easily manipulated than skyrmions. If the above expectations are true, they will be also candidates for the spintronics devices and, moreover, more beneficial than the skyrmions from the viewpoint of manipulation using the electrical current. In or- der to confirm the above expectations on skyrmioniums, we have investigated about dynamics of skyrmioniums using micromagnetic simulations and presented in SSDM2019. ${ }^{12)}$ In this paper, as a further investigation about skyrmioniums, we provide not only advantages but also disadvantages for applying skyrmioniums to the spintronics devices and, moreover, provide how we can generate skyrmioniums.

\section{Methods}

Magnetization of skyrmionic structures such as skyrmions and skyrmioniums are described by the spherical coordinates as follows:

$$
\boldsymbol{m}(\boldsymbol{r})=(\cos \Phi(\phi) \sin \theta(r), \sin \Phi(\phi) \sin \theta(r), \cos \theta(r)),
$$

where $\boldsymbol{m}$ is the magnetization unit vector and $\Phi(\phi)=n \phi+\gamma$. Here, $n$ is the vorticity and $\gamma$ is the helicity. In this study, we consider the structure with $n=1$ and $\gamma=0$ or $\gamma=\pi$. In order to obtain skyrmionic structures, we calculate the lowest energy of the magnetization structures given by Eq. (1) using a variational principle. ${ }^{8,13-18)}$ In this study, a total energy is composed of the exchange energy, the uniaxial perpendicular anisotropy energy, the interfacial Dzyaloshinskii-Moriya interaction (DMI) energy, and the demagnetization energy. Thus, the total energy density is written as follows:

$$
\begin{aligned}
\varepsilon= & A\left[\left(\frac{\partial \boldsymbol{m}}{\partial x}\right)^{2}+\left(\frac{\partial \boldsymbol{m}}{\partial y}\right)^{2}+\left(\frac{\partial \boldsymbol{m}}{\partial z}\right)^{2}\right]+K\left(1-m_{z}^{2}\right) \\
& +D_{\mathrm{DMI}}\left[m_{z}\left(\frac{\partial m_{x}}{\partial x}+\frac{\partial m_{x}}{\partial y}\right)-\left(m_{x} \frac{\partial m_{z}}{\partial x}+m_{y} \frac{\partial m_{z}}{\partial y}\right)\right] \\
& +\frac{1}{2} \mu_{0} M_{s} \boldsymbol{m} \cdot \boldsymbol{H}_{\mathrm{d}}
\end{aligned}
$$

where, $A$ is the exchange stiffness, $K$ is the perpendicular anisotropy constant, $D_{\mathrm{DMI}}$ is the DMI constant, $\mu_{0}$ is the vacuum permeability, $M_{s}$ is the saturation magnetization, and $\boldsymbol{H}_{\mathrm{d}}$ is the demagnetization field. In order to obtain the demagnetization field, we use the local approximation. Namely, the demagnetization field is given by $\boldsymbol{H}_{\mathrm{d}}=-M_{s} m_{z} \hat{z}$. For obtaining skyrmion structures, it is imposed that the boundary condition $\theta(0)=\pi$ and $\theta(\infty)=0$, or $\theta(0)=0$ and $\theta(\infty)=\pi$. Under these boundary conditions, we can obtain $\Phi(\phi)=\phi$ or $\Phi(\phi)=\phi+\pi$, respectively. On the other hand, for calculating a 
skyrmionium structure, it is imposed that the boundary condition $\theta(0)=2 \pi$ and $\theta(\infty)=0$. Under this boundary condition, we can obtain $\Phi(\phi)=\phi$. As a result, the Euler-Lagrange equation of the angle of $\theta$ is obtained as follows:

$$
\frac{d^{2} \theta}{d \tilde{r}^{2}}+\frac{1}{\tilde{r}} \frac{d \theta}{d \tilde{r}}-\frac{\sin \theta \cos \theta}{\tilde{r}} \pm \frac{D_{\mathrm{DMI}}}{\sqrt{A K}} \frac{\sin ^{2} \theta}{\tilde{r}}-\sin \theta \cos \theta=0,
$$

where $\tilde{r}=r \sqrt{K / A}$. The sign of the fourth term is plus when the boundary condition is $\theta(0)=\pi$ and $\theta(\infty)=0$, or $\theta(0)=2 \pi$ and $\theta(\infty)=0$. On the other hand, the above sign is minus when the boundary condition is $\theta(0)=0$ and $\theta(\infty)=\pi$. Substituting the solution of Eq. (3) into Eq.(1), magnetization structures can be obtained and they are identified by the topological invariant called the topological charge which is defined by

$$
Q=\frac{1}{4 \pi} \int \boldsymbol{m} \cdot\left(\frac{\partial \boldsymbol{m}}{\partial x} \times \frac{\partial \boldsymbol{m}}{\partial y}\right) d x d y,
$$

where $Q$ corresponds to how many times magnetization wraps the $S^{2}$ sphere. Substituting Eq. (1) into Eq. (4), the topological charges of the skyrmionic structures are explicitly written as follows:

$$
Q=\frac{n}{2}\left(m_{z}(0)-m_{z}(\infty)\right)
$$

Thus, the topological charges are $Q= \pm 1$ or $Q=0$ in the cases of skyrmions or skyrmioniums, respectively.

When spin torques work on magnetization, magnetization dynamics is described by the following modified LandauLifshitz-Gilbert equation: ${ }^{2,19-31)}$

$$
\frac{d \boldsymbol{m}}{d t}=-\gamma \mu_{0} \boldsymbol{m} \times \boldsymbol{H}_{\mathrm{eff}}+\alpha \boldsymbol{m} \times \frac{d \boldsymbol{m}}{d t}+\frac{\boldsymbol{\tau}}{M_{s}},
$$

where $\gamma$ is the gyromagnetic ratio, $\alpha$ is the Gilbert damping constant, $\tau$ is the spin torque working on the magnetization, and $\boldsymbol{H}_{\text {eff }}$ is the effective magnetic field which represents all the effects working on magnetic moments and described by the functional derivative as follows:

$$
\boldsymbol{H}_{\mathrm{eff}}=-\frac{1}{\mu_{0}} \frac{\delta E[\boldsymbol{M}]}{\delta \boldsymbol{M}},
$$

where $E$ is the total energy of all the effects working on magnetic moments and $\boldsymbol{M}$ is the magnetization vector. When spin transfer torque (STT) works on magnetization, $\tau$ is given by ${ }^{20}$ )

$$
\boldsymbol{\tau}=\frac{\gamma \hbar P}{2 e M_{S}\left(1+\beta^{2}\right)}[(\boldsymbol{j} \cdot \nabla) \boldsymbol{m}-\beta \boldsymbol{m} \times(\boldsymbol{j} \cdot \nabla) \boldsymbol{m}],
$$

where $\hbar$ is the Dirac constant, $P$ is the spin polarization, $e$ is the elementary charge, $\boldsymbol{j}$ is the current density, and $\beta$ is the non-adiabatic constant of STT. In order to solve Eq. (6) numerically, we employ a finite element method (FEM) for space discretization and a finite difference method (FDM) based on the midpoint rule for time discretization. ${ }^{32,33)}$ Firstly, in order to calculate the effective magnetic field by utilizing FEM, we transform Eq. (7) to the following equation:

$$
\frac{\delta}{\delta \boldsymbol{M}}\left(E[\boldsymbol{M}]+\mu_{0} \boldsymbol{M} \cdot \boldsymbol{H}_{\mathrm{eff}}\right)=0
$$

For solving Eq. (9), we utilize the Rayleigh-Ritz method. When utilizing this method for calculating the effective magnetic field, we need not to take into consideration the following boundary condition explicitly. ${ }^{34)}$

$$
\frac{d \boldsymbol{m}}{d n}=\frac{D_{\mathrm{DMI}}}{2 A}(\hat{z} \times \boldsymbol{n}) \times \boldsymbol{m},
$$

where $\boldsymbol{n}$ is the unit vector perpendicular to the edge. This is because the process of solving Eq. (9) includes the above boundary condition naturally. Therefore, when utilizing the Rayleigh-Ritz method, there exists a benefit that we can equally treat all elements without dealing with the edge exceptionally. Next, in order to obtain time evolution of magnetization using FDM based on the midpoint rule, we substitute Eq. (8) into Eq. (6) and rearrange Eq. (6) to the following form:

$$
\frac{d \boldsymbol{m}}{d t}=-\frac{1}{1+\alpha^{2}} \gamma \mu_{0} \boldsymbol{m} \times \tilde{\boldsymbol{H}},
$$

where

$$
\begin{aligned}
& \tilde{\boldsymbol{H}}=\boldsymbol{H}+\alpha \boldsymbol{m} \times \boldsymbol{H} \\
& \boldsymbol{H}=\boldsymbol{H}_{\mathrm{eff}}+\frac{\hbar P}{2 e \mu_{0} M_{s}^{2}\left(1+\beta^{2}\right)}[\boldsymbol{m} \times(\boldsymbol{j} \cdot \nabla) \boldsymbol{m}+\beta(\boldsymbol{j} \cdot \nabla) \boldsymbol{m}] .
\end{aligned}
$$

For time discretization of Eq. (11), the midpoint rule approximations are introduced as follows:

$$
\begin{aligned}
\left(\frac{d \boldsymbol{m}}{d t}\right)^{\left(n+\frac{1}{2}\right)} & =\frac{\boldsymbol{m}^{(n+1)}-\boldsymbol{m}^{(n)}}{\Delta t}+O\left(\Delta t^{2}\right), \\
\boldsymbol{m}^{\left(n+\frac{1}{2}\right)} & =\frac{\boldsymbol{m}^{(n+1)}+\boldsymbol{m}^{(n)}}{2}+O\left(\Delta t^{2}\right),
\end{aligned}
$$

where superscript $(n)$ represents the time step $t_{n}$ and $\Delta t$ is the time interval $t_{n+1}-t_{n}$. Moreover, we calculate $\tilde{\boldsymbol{H}}$ by an extrapolation method as follows:

$$
\tilde{\boldsymbol{H}}^{\left(n+\frac{1}{2}\right)}=\frac{3}{2} \tilde{\boldsymbol{H}}^{(n)}-\frac{1}{2} \tilde{\boldsymbol{H}}^{(n-1)}+O\left(\Delta t^{2}\right) .
$$

By substituting Eqs. (14), (15), and (16) into Eq. (11) and neglecting $O\left(\Delta t^{2}\right)$, we obtain a time-discretized LLG equation as follows:

$$
\begin{aligned}
\boldsymbol{m}^{(n+1)}-\boldsymbol{m}^{(n)}=- & \frac{\gamma \mu_{0} \Delta t}{2\left(1+\alpha^{2}\right)}\left(\boldsymbol{m}^{(n+1)}+\boldsymbol{m}^{(n)}\right) \\
& \times\left(\frac{3}{2} \tilde{\boldsymbol{H}}^{(n)}-\frac{1}{2} \tilde{\boldsymbol{H}}^{(n-1)}\right) .
\end{aligned}
$$

Since Eq. (17) is a linear vector equation for $\boldsymbol{m}^{(n+1)}$, the value of $\boldsymbol{m}^{(n+1)}$ is calculated easily. Moreover, when obtaining next step magnetization using Eq. (17), there exist a desirable property that magnitude of the magnetization unit vector is conserved at any time steps. This is because we can obtain the following equation by calculating an inner product of both sides of Eq. (17) with the vector $\boldsymbol{m}^{(n+1)}+\boldsymbol{m}^{(n)}$ :

$$
\left|\boldsymbol{m}^{(n+1)}\right|^{2}=\left|\boldsymbol{m}^{(n)}\right|^{2} \text {. }
$$

After discretizing the space and the time by utilizing the above methods, the micromagnetic simulation can be performed by solving Eq. (17).

Generally, it is necessary to solve the LLG equation numerically. However, under the special condition, there exist analytical solutions described by the Thiele equation which governs the steady state motion of magnetization. ${ }^{35)}$ The Thiele equation is derived from the LLG equation if it is assumed that 
configuration of magnetization does not change and only translational motion is possible in magnetization dynamics. Especially, from Eqs. (6) and (8), we can obtain the Thiele equation which describes the steady state motion of a skyrmionic structure effected by STT as follows:

$$
\begin{aligned}
\boldsymbol{G} \times & \left(\boldsymbol{v}-\frac{\gamma \hbar P}{2 e M_{S}\left(1+\beta^{2}\right)} \boldsymbol{j}\right) \\
& -4 \pi \alpha \boldsymbol{D} \cdot\left(\boldsymbol{v}-\frac{\gamma \hbar P}{2 e M_{S}\left(1+\beta^{2}\right)} \frac{\beta}{\alpha} \boldsymbol{j}\right)=0,
\end{aligned}
$$

where $\boldsymbol{G}=(0,0,-4 \pi Q)$ is the gyromagnetic coupling vector, $\boldsymbol{v}$ is the velocity of skyrmionic structures, $\boldsymbol{D}=\left(\begin{array}{ll}D_{x x} & D_{x y} \\ D_{y x} & D_{y y}\end{array}\right)$ is the dissipative force tensor. Here, the components of this tensor $\boldsymbol{D}$ are determined by configuration of a skyrmionic structure and generally written as follows: ${ }^{35)}$

$$
D_{i j}=\frac{1}{4 \pi} \int \frac{\partial \boldsymbol{m}}{\partial x_{i}} \cdot \frac{\partial \boldsymbol{m}}{\partial x_{j}} d x d y .
$$

In the cases of a skyrmionic structure, by substituting Eq. (1) into Eq. (20), the components of $\boldsymbol{D}$ is given by

$$
\begin{aligned}
& D_{x x}=D_{y y}=D, \\
& D_{x y}=D_{x y}=0,
\end{aligned}
$$

where

$$
D=\frac{1}{4} \int_{0}^{\infty}\left[\left(\frac{d \theta}{d r}\right)^{2}+\frac{n^{2} \sin ^{2} \theta}{r^{2}}\right] r d r,
$$

and $n$ is the vorticity. Using Eq. (22), we can obtain the velocity of the skyrmionic structures in the case that the current density is spatially homogeneous and has only $x$-component $\boldsymbol{j}=\left(j_{x}, 0\right)$ as follows:

$$
\begin{aligned}
& v_{x}=\frac{-Q^{2}-\alpha \beta D^{2}}{Q^{2}+\alpha^{2} D^{2}} \frac{\gamma \hbar P}{2 e M_{S}\left(1+\beta^{2}\right)} j_{x}, \\
& v_{y}=\frac{Q D(\beta-\alpha)}{Q^{2}+\alpha^{2} D^{2}} \frac{\gamma \hbar P}{2 e M_{s}\left(1+\beta^{2}\right)} j_{x} .
\end{aligned}
$$

Since the topological charges of skyrmions are $Q= \pm 1$, they have the perpendicular velocity components to the electrical current flow under the condition of $\alpha \neq \beta$. On the other hand, since the topological charges of skyrmioniums are always $Q=0$, their motion is always parallel with the current flow. These solutions are true only if the above assumptions hold. However, in real materials, the magnetic inner structures frequently change while moving. In our previous study, ${ }^{4)}$ it is found that there is a discrepancy between the numerical solutions by the LLG and the analytical solutions by the Thiele equation. Therefore, we need to solve the LLG equation to compare their solutions.

\section{Results and discussion}

In micromagnetic simulation, the ferromagnetic material is assumed to be cobalt thin film on platinum and we utilize the following material parameters: ${ }^{36)} M_{s}=580 \mathrm{kA} / \mathrm{m}$, $A=15 \mathrm{pJ} / \mathrm{m}, D=3.5 \mathrm{~mJ} / \mathrm{m}^{2}, K=0.8 \mathrm{MJ} / \mathrm{m}^{3}, \beta=0.4$, $P=40 \%$, and $\alpha=0.2$ or $\alpha=0.02$. First of all, we obtain the angles $\theta(r)$ of skyrmionic structures by solving Eq. (3) and the solutions are shown in Fig. 1(a). By substituting the above solutions into Eq. (1), the structures of the skyrmion (a)

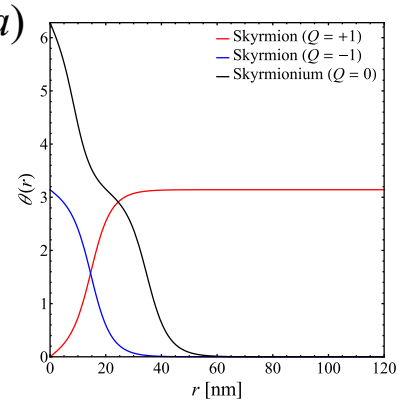

(c)

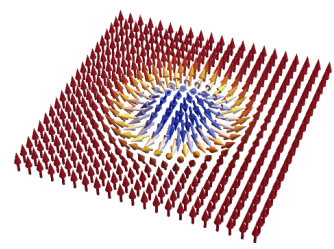

(b)

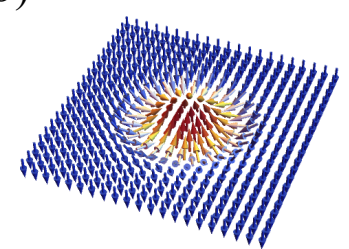

(d)

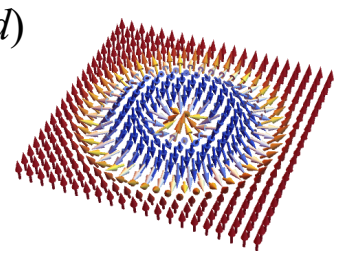

Fig. 1. (a) The angles of $\theta(r)$ obtained using the variational method. The red, blue, and black lines represents $\theta(r)$ of the $Q=+1$ skyrmion, the $Q=-1$ skyrmion, and the skyrmionium, respectively. Magnetization structures of $(b)$ the $Q=+1$ skyrmion, $(c)$ the $Q=-1$ skyrmion, and $(d)$ the skyrmionium obtained using the above $\theta(r)$, respectively. In figures $(b)$, $(c)$, and $(d)$, the directions and the colors of arrows represent the magnetization-direction and the magnitude of $z$-component of magnetization at each point, respectively.

with $Q=+1$, the skyrmion with $Q=-1$, and the skyrmionium can be obtained as shown in Figs. 1(b), 1(c), and 1(d), respectively. Here, the above skyrmionic structures are called the Néel-type since the magnetization changes like Néel-type domain walls as we go away from the center of their structures. Moreover, these figures show the fact that the skyrmionium is equivalently composed of the two skyrmions with the topological charges $Q$ of \pm 1 . In order to obtain sizes of the skyrmionic structures, we define a radius $r_{0}$ where the condition of $m_{z}\left(r_{0}\right)=0$ is satisfied. As a result, the radii of both the skyrmions are $14.54 \mathrm{~nm}$ and the radius of the skyrmionium is $34.61 \mathrm{~nm}$. This result implies that it is less beneficial to utilize the skyrmionium in order to represent one bit of information than the case of the skyrmion from the viewpoint of density of information. On the other hand, the skyrmionium also has an advantage for the spintronics devices. Substituting the solution of Eq. (3) into Eq. (22), we obtain the value of $D$ of 1.73 and 5.07 for the skyrmions and the skyrmionium, respectively. By utilizing these values, we can obtain velocities of the skyrmionic structures using equation (23). Table $\mathrm{I}(a)$ shows the velocities when the spin polarized current density of $-1 \mathrm{TA} / \mathrm{m}^{2}$ is applied in the $x$-direction. As shown in Table $\mathrm{I}(a)$, the speed of the skyrmionium is faster than that of the skyrmions. This becomes the advantage for the spintronics devices.

Next, we perform micromagnetic simulations to confirm that the above result realizes in the real magnetic structures. For the simulations, we utilize the skyrmionic structures obtained by solving Eq. (3) for the initial states and apply the spin polarized current density of $-1 \mathrm{TA} / \mathrm{m}^{2}$ in the $x$-direction. Here, in the micromagnetic simulations, we set the time steps to be $10 \mathrm{fs}$ and $5 \mathrm{fs}$ in the cases of $\alpha=0.2$ and $\alpha=0.02$, respectively. Figure 2 shows the time evolution of the skyrmions and the skyrmionium motion. As shown in Figs. 2(a) and 
(a)

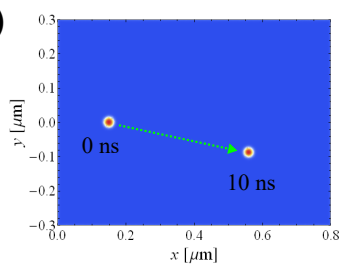

(b)

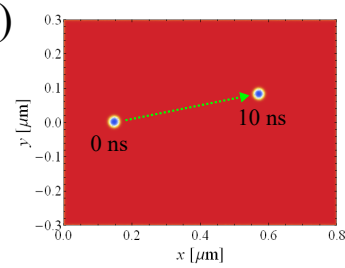

$(c)$

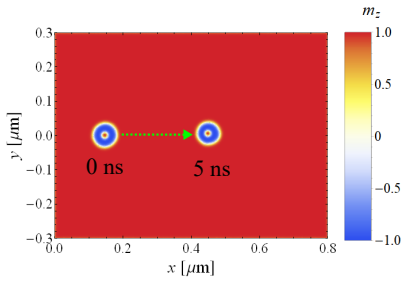

Fig. 2. The time evolution of (a) the $Q=+1$ skyrmion motion, $(b)$ the $Q=-1$ skyrmion motion, and $(c)$ the skyrmionium motion in the case of $\alpha=0.2$.

(a)

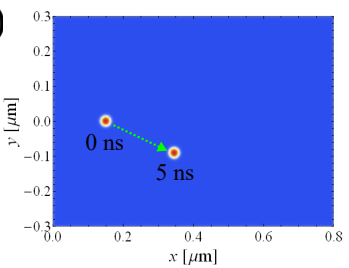

(b)

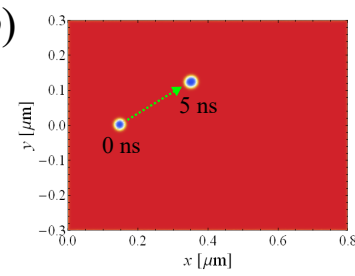

(c)

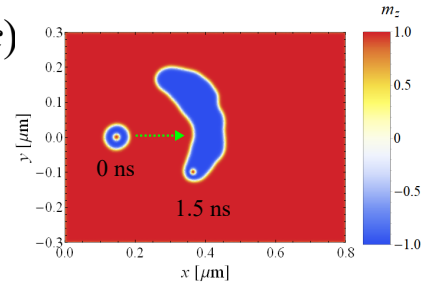

Fig. 3. The time evolution of ( $a$ ) the $Q=+1$ skyrmion motion, $(b)$ the $Q=-1$ skyrmion motion, and (c) the skyrmionium motion in the case of $\alpha=0.02$.

Table I. (a) Velocities of the skyrmionic structures obtained by solving the Thiele equation and $(b)$ average velocities of the skyrmionic structures by the micromagnetic simulation in both the cases of $\alpha=0.2$ and $\alpha=0.02$.

(a)

\begin{tabular}{|c|c|c|c|}
\hline & $v_{x}$ & $v_{y}$ & $|v|$ \\
\hline \multicolumn{4}{|l|}{$\alpha=0.2$} \\
\hline Skyrmion $(Q=+1)$ & $38.15 \mathrm{~m} / \mathrm{s}$ & $-10.67 \mathrm{~m} / \mathrm{s}$ & $39.62 \mathrm{~m} / \mathrm{s}$ \\
\hline Skyrmion $(Q=-1)$ & $38.15 \mathrm{~m} / \mathrm{s}$ & $10.67 \mathrm{~m} / \mathrm{s}$ & $39.62 \mathrm{~m} / \mathrm{s}$ \\
\hline Skyrmionium $(Q=0)$ & $68.91 \mathrm{~m} / \mathrm{s}$ & $0.00 \mathrm{~m} / \mathrm{s}$ & $68.91 \mathrm{~m} / \mathrm{s}$ \\
\hline \multicolumn{4}{|l|}{$\alpha=0.02$} \\
\hline Skyrmion $(Q=+1)$ & $35.24 \mathrm{~m} / \mathrm{s}$ & $-22.68 \mathrm{~m} / \mathrm{s}$ & $41.91 \mathrm{~m} / \mathrm{s}$ \\
\hline $\operatorname{Skyrmion}(Q=-1)$ & $35.24 \mathrm{~m} / \mathrm{s}$ & $22.68 \mathrm{~m} / \mathrm{s}$ & $41.91 \mathrm{~m} / \mathrm{s}$ \\
\hline Skyrmionium $(Q=0)$ & $689.1 \mathrm{~m} / \mathrm{s}$ & $0.00 \mathrm{~m} / \mathrm{s}$ & $689.1 \mathrm{~m} / \mathrm{s}$ \\
\hline
\end{tabular}

(b)

\begin{tabular}{l|rrr} 
& \multicolumn{1}{|c}{$v_{x}$} & $|v|$ \\
\hline$\alpha=0.2$ & & & \\
Skyrmion $(Q=+1)$ & $40.93 \mathrm{~m} / \mathrm{s}$ & $-8.74 \mathrm{~m} / \mathrm{s}$ & $41.85 \mathrm{~m} / \mathrm{s}$ \\
Skyrmion $(Q=-1)$ & $42.39 \mathrm{~m} / \mathrm{s}$ & $8.17 \mathrm{~m} / \mathrm{s}$ & $43.16 \mathrm{~m} / \mathrm{s}$ \\
Skyrmionium $(Q=0)$ & $60.66 \mathrm{~m} / \mathrm{s}$ & $1.04 \mathrm{~m} / \mathrm{s}$ & $60.67 \mathrm{~m} / \mathrm{s}$ \\
\hline$\alpha=0.02$ & & \\
Skyrmion $(Q=+1)$ & $38.90 \mathrm{~m} / \mathrm{s}$ & $-18.06 \mathrm{~m} / \mathrm{s}$ & $42.88 \mathrm{~m} / \mathrm{s}$ \\
Skyrmion $(Q=-1)$ & $40.69 \mathrm{~m} / \mathrm{s}$ & $24.54 \mathrm{~m} / \mathrm{s}$ & $47.52 \mathrm{~m} / \mathrm{s}$ \\
Skyrmionium $(Q=0)$ & $152.7 \mathrm{~m} / \mathrm{s}$ & $37.05 \mathrm{~m} / \mathrm{s}$ & $157.1 \mathrm{~m} / \mathrm{s}$
\end{tabular}

2(b), similarly to the result of the Thiele equation, the motion of the skyrmions is not parallel with the direction of the spin polarized current and they deflect to the right or left directions depending on the sign of their topological charges. On the other hand, in the case of the skyrmionium, the motion is parallel with the direction of the spin polarized current. Therefore, the skyrmionium can be more easily manipulated than the skyrmions. Although the above results can be also obtained by the Thiele equation, there exists a discrepancy between the velocities obtained by the Thiele equation and those obtained using the micromagnetic simulation as shown in Tables $\mathrm{I}(a)$ and $\mathrm{I}(b)$ in both the cases of $\alpha=0.2$ and 0.02 . As shown in these tables, the speed of the skyrmionic structures in the micromagnetic simulation is different from that obtained by the Thiele equation. This is because the real motion does not have constant velocity and the velocities of the real motions are those obtained by averaging the fluctuating velocities according to the change of micromagnetic internal structures.

The discrepancy between the results of the above two methods is more serious with decreasing value of the Gilbert damping constant $\alpha$. This is because the skyrmionic structures distorts largely when $\alpha$ is very small and the value of the components of the dissipative force tensor in Eq. (20) can not remain constant. Namely, the system does not satisfy the assumption that configuration of magnetization does not change and only translational motion is possible in magnetization dynamics. Figure 3 shows the time evolution of the motion of the skyrmionic structures in the case of $\alpha=0.02$. Although the structures of the skyrmions do not distort as shown in Figs. 3(a) and 3(b), the structure of the skyrmionium distorts largely as shown in Fig. 3(c). In the case of the skyrmionium, among two skyrmions making skyrmionium, inner skyrmion with the topological charge $Q$ of +1 and the outer skyrmion with the topological charge $Q$ of -1 start to deflect to the right and left direction, respectively, due to rapid precession of internal structures when the Gilbert damping constant becomes very small. Therefore, the magnetic structures of skyrmionium start to collapse and transform to elliptic shape. Namely, the rigidity of the skyrmionium disappears in the case of very small $\alpha$. Now, the skyrmionium distortion can be estimated by the value of the dissipative force tensor as shown in Fig. 4. We notice that $D_{x x}$ becomes large with the time elapsing from Fig. 4. The following is the reason why $D_{x x}$ becomes large with the time elapsing. Here, we consider the influence of distortion of magnetic structures on the dissipative force tensor $\boldsymbol{D}$. The main distortion of magnetic structures is assumed to be scaling where scaling factors are $a$ along $x$-direction and $b$ along $y$-direction, respectively. Furthermore, for other distortions, we incorporate their effects as the random distortion $\delta \boldsymbol{m}(x, y)$. Then, the distorted magnetic distributions is given by

$$
\tilde{\boldsymbol{m}}(x, y)=\boldsymbol{m}\left(\frac{x}{a}, \frac{y}{b}\right)+\delta \boldsymbol{m}(x, y),
$$

where $\boldsymbol{m}$ is the skyrmionium magnetization described by 


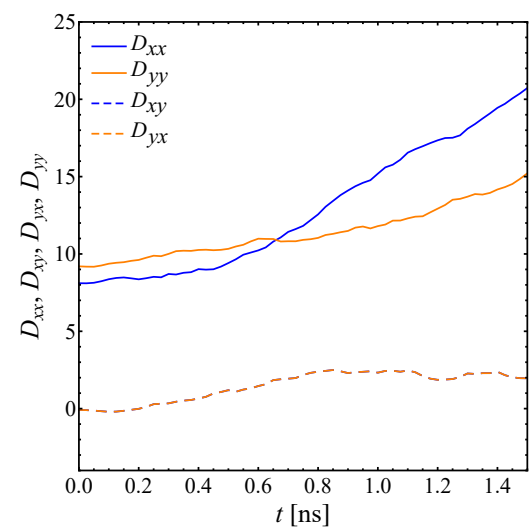

Fig. 4. The time dependence of the components of the dissipative force tensor of the skyrmionium in the case of $\alpha=0.02$. $D_{x y}$ and $D_{y x}$ have the same values.

Eq. (1). Substituting Eq. (24) into Eq. (20), we obtain the components of the dissipative force tensor $\boldsymbol{D}$ of the distorted skyrmionium as follows:

$$
\begin{aligned}
& D_{x x}=\frac{b}{a} D+\frac{1}{4 \pi} \int\left[2 \frac{\partial \boldsymbol{m}(x / a, y / b)}{\partial x} \cdot \frac{\partial \delta \boldsymbol{m}(x, y)}{\partial x}\right. \\
& \left.+\left(\frac{\partial \delta \boldsymbol{m}(x, y)}{\partial x}\right)^{2}\right] d x d y \\
& D_{y y}=\frac{a}{b} D+\frac{1}{4 \pi} \int\left[2 \frac{\partial \boldsymbol{m}(x / a, y / b)}{\partial y} \cdot \frac{\partial \delta \boldsymbol{m}(x, y)}{\partial y}\right. \\
& \left.+\left(\frac{\partial \delta \boldsymbol{m}(x, y)}{\partial y}\right)^{2}\right] d x d y \\
& D_{x y}=D_{y x}=\frac{1}{4 \pi} \int\left(\frac{\partial \boldsymbol{m}(x / a, y / b)}{\partial x} \cdot \frac{\partial \delta \boldsymbol{m}(x, y)}{\partial y}\right. \\
& +\frac{\partial \boldsymbol{m}(x / a, y / b)}{\partial y} \cdot \frac{\partial \delta \boldsymbol{m}(x, y)}{\partial x} \\
& \left.+\frac{\partial \delta \boldsymbol{m}(x, y)}{\partial x} \cdot \frac{\partial \delta \boldsymbol{m}(x, y)}{\partial y}\right) d x d y,
\end{aligned}
$$

where $D$ is the diagonal component of $\boldsymbol{D}$ of the non-distorted skyrmionium described by Eq. (22). In order to estimate the value of $\frac{b}{a}$, we consider that the integral including the linear terms of the random distortion is canceled out due to the randomness. On the other hand, the integral including the quadratic terms of the random distortion in Eq. (25) and (26) can not be canceled out and increase both the value of $D_{x x}$ and $D_{y y}$. At a glance, from Fig. 4 , it is thought that $D_{y y}$ is expected to decrease with the time elapsing since the behavior of $D_{x x}$ shows that $b / a$ increases with the time elapsing. However, it is considered that the contribution of the quadratic terms to the integral overcomes the decrease of $D_{y y}$ and makes the increase of $D_{x x}$ overestimated. In order to estimate the skyrmionium distortion, neglecting the quadratic terms, we approximate the ratio between the scaling factors $a$ and $b$ by utilizing the overestimated value of $D_{x x}$ as follows:

$$
\frac{b}{a} \approx \frac{D_{x x}}{D} \text {. }
$$

As a result, using the time dependence of $D_{x x}$ as shown in Fig. 4 and the value of $D=5.07$ which is calculated by utiliz-

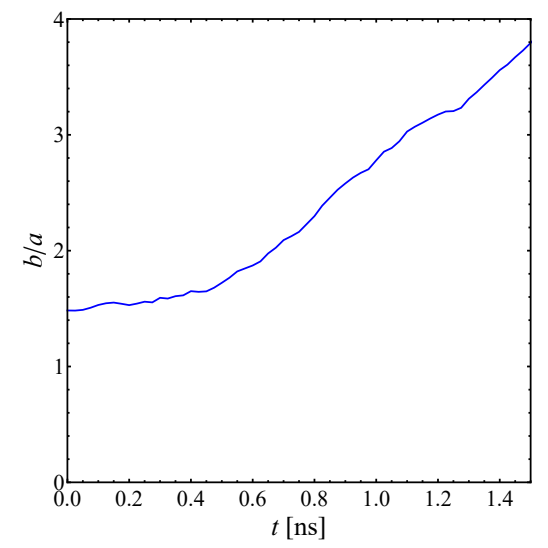

Fig. 5. The time dependence of the ratio between scaling factor $a$ along $x$-direction and scaling factor $b$ along $y$-direction in the case of $\alpha=0.02$.
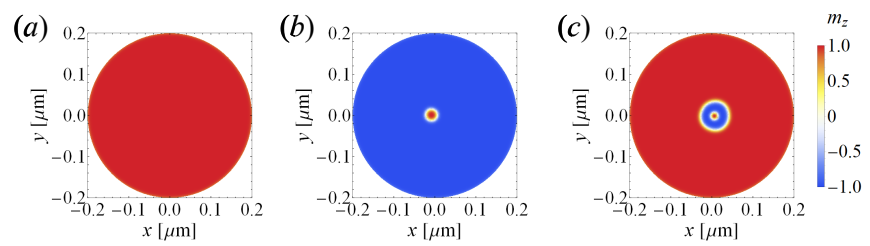

Fig. 6. Snap shots of the process of creating a skyrmionium. (a) The initial state. (b) The state after applying a pulsed magnetic field to the initial state and relaxing. (c) The state after applying a pulsed magnetic field to the state of $(b)$ and relaxing.

ing the solution of Eq. (3), we can obtain the time dependence of the ratio between the scaling factors $a$ and $b$ as shown in Fig. 5. From this figure, it is found that the skyrmionium becomes elliptic shape with time elapsing in the micromagnetic simulation. Moreover, we can approximate the above ratio by a quadratic function as follows:

$$
\frac{b}{a}=0.774 t^{2}+0.503 t+1.394
$$

Although, so far, we have discussed the advantages and the disadvantages when applying skyrmioniums to the spintronics devices, here, we are going to provide how to create a skyrmionium by performing a micromagnetic simulation since there exists few experimental studies on observations and creating methods of a skyrmionium to our knowledge. In our method, we can create the skyrmionium in a ferromagnetic disk by utilizing pulses of magnetic fields. Firstly, we prepare the ferromagnetic disk for an initial state as shown in Fig. 6(a). Then, we have applied the magnetic field of $-0.8 \mathrm{MA} / \mathrm{m}(\approx-1.01 \mathrm{~T})$ in the $z$-direction until $0.2 \mathrm{~ns}$ and relaxed until $2 \mathrm{~ns}$. As a result, a skyrmion can be obtained at the center of the region as shown in Fig. 6(b). After that, we have applied the magnetic field of $0.8 \mathrm{MA} / \mathrm{m}(\approx 1.01 \mathrm{~T})$ in the $z$-direction during $0.08 \mathrm{~ns}$ and relaxed during $4.5 \mathrm{~ns}$. Then, we can obtain a skyrmionium in the center of the region as shown in Fig. 6(c).

\section{Conclusions}

In this study, we have discussed the advantages and the disadvantages when applying skyrmioniums to the spintronics devices in comparison with the case of the skyrmions. Although the skyrmioniums have the vortex-like structures as 
the same as the skyrmions, sizes of the skyrmioniums are larger than those of the skyrmions. Therefore, it is less beneficial to utilize the skyrmionium in order to represent one bit of information than the case of the skyrmion from the viewpoint of density of information. However, from the viewpoint of the manipulation, the skyrmioniums are more beneficial than the skyrmions. This is because it is derived from the Thiele equation that the motion of the skyrmioniums is always parallel with the direction of the electrical current flow. Namely, the skyrmioniums can be more easily manipulated than the skyrmions. Moreover, we have found that the speed of the skyrmionium motion is faster than that of the skyrmion motion. These results of the skyrmionium dynamics can be also verified by utilizing the micromagnetic simulations in the case of the relatively large Gilbert damping constant. On the other hand, when the Gilbert damping constant is relatively small, it is found that there is no benefit to utilize the skyrmionium since the skyrmionium structure distorts largely. From the above reason, the skyrmioniums are not entirely more beneficial to the spintronics devices than the skyrmions. In the last of the section 3 , we have provided a creation method of the skyrmionium by utilizing pulsed magnetic field. We hope that this is useful to create and investigate the skyrmioniums experimentally.

\section{Acknowledgment}

This work is partially supported by a Grant-in-Aid for Scientific Research (Grant No. 16K04872, Grant No.17H03225 ) from JSPS, Center for Spintronics Research Network (CSRN) Tohoku University, and Dynamic Alliance for Open Innovation Bridging Human, Environment and Materials.

1) A. Fert, V. Cros, and J. Sampaio, Nat. Nanotechnol. 8, 152 (2013).

2) W. Jiang, G. Chen, K. Liu, J. Zang, S. G. t. Velthuis, and A. Hoffmann, Phys. Rep. 704, 1 (2017).

3) N. Nagaosa and Y. Tokura, Nat. Nanotechnol. 8, 899 (2013).

4) Y. Ishida and K. Kondo, J. Magn. Magn. Mater. 493, 165687 (2020).

5) W. Jiang, P. Upadhyaya, W. Zhang, G. Yu, M. B. Jungfleisch, F. Y. Fradin, J. E. Pearson, Y. Tserkovnyak, K. L. Wang, O. Heinonen, S. G. E. t. Velthuis, and A. Hoffmann, Science 349, 283 (2015).

6) W. Jiang, X. Zhang, G. Yu, W. Zhang, X. Wang, M. Benjamin Jungfleisch, J. E. Pearson, X. Cheng, O. Heinonen, K. L. Wang, Y. Zhou, A. Hoffmann, and S. G. E. t. Velthuis, Nat. Phys. 13, 162 (2016).

7) K. Litzius, I. Lemesh, B. Krüger, P. Bassirian, L. Caretta, K. Richter, F. Büttner, K. Sato, O. A. Tretiakov, J. Förster, R. M. Reeve, M. Weigand, I. Bykova, H. Stoll, G. Schütz, G. S. D. Beach, and M. Kläui, Nat. Phys. 13, 170 (2016).

8) A. Bogdanov and A. Hubert, J. Magn. Magn. Mater. 195, 182 (1999).

9) M. Finazzi, M. Savoini, A. R. Khorsand, A. Tsukamoto, A. Itoh, L. Duò, A. Kirilyuk, T. Rasing, and M. Ezawa, Phys. Rev. Lett. 110, 177205 (2013).

10) S. Zhang, F. Kronast, G. v. d. Laan, and T. Hesjedal, Nano Lett. 18, 1057 (2018).

11) H. Fujita and M. Sato, Phys. Rev. B 95, 054421 (2017).

12) Y. Ishida and K. Kondo, Extended Abstracts of SSDM 2019, 945 (2019).

13) A. Bogdanov and A. Hubert, J. Magn. Magn. Mater. 138, 255 (1994).

14) A. Bogdanov and A. Hubert, Phys. Status Solidi B 186, 527 (1994).

15) A. N. Bogdanov and U. K. Rößler, Phys. Rev. Lett. 87, 037203 (2001).

16) A. Bogdanov and D. Yablonskii, J. Exp. Theor. Phys. 95, 178 (1989).

17) N. Romming, A. Kubetzka, C. Hanneken, K. v. Bergmann, and R. Wiesendanger, Phys. Rev. Lett. 114, 177203 (2015).

18) N. S. Kiselev, A. N. Bogdanov, R. Schäfer, and U. K. Rößler, J. Phys. D: Appl. Phys. 44, 392001 (2011).
19) T. L. Gilbert, IEEE Trans. Magn. 40, 3443 (2004).

20) R. Tomasello, E. Martinez, R. Zivieri, L. Torres, M. Carpentieri, and G. Finocchio, Sci. Rep. 4, 6784 (2014).

21) W. Kang, C. Zheng, Y. Huang, X. Zhang, Y. Zhou, W. Lv, and W. Zhao, IEEE Electron Device Lett. 37, 924 (2016).

22) M.-W. Yoo, V. Cros, and J.-V. Kim, Phys. Rev. B 95, 184423 (2017).

23) V. Raposo, R. F. Luis Martinez, and E. Martinez, AIP Adv. 7, 056017 (2017).

24) S.-Z. Lin, C. Reichhardt, C. D. Batista, and A. Saxena, Phys. Rev. Lett. 110, 207202 (2013)

25) W. Koshibae and N. Nagaosa, Sci. Rep. 9, 5111 (2019).

26) P. Upadhyaya, G. Yu, P. K. Amiri, and K. L. Wang, Phys. Rev. B 92, 134411 (2015).

27) C. Schütte, J. Iwasaki, A. Rosch, and N. Nagaosa, Phys. Rev. B 90, 174434 (2014).

28) W. Koshibae, Y. Kaneko, J. Iwasaki, M. Kawasaki, Y. Tokura, and N. Nagaosa, Jpn. J. Appl. Phys. 54, 053001 (2015).

29) S. Komineas and N. Papanicolaou, Phys. Rev. B 92, 174405 (2015).

30) X. Zhang, G. P. Zhao, H. Fangohr, J. P. Liu, W. X. Xia, J. Xia, and F. J. Morvan, Sci. Rep. 5, 7643 (2015).

31) J. Iwasaki, M. Mochizuki, and N. Nagaosa, Nat. Commun. 4, 1463 (2013).

32) O. Bottauscio, M. Chiampi, and A. Manzin 44, 3149 (2008).

33) C. Serpico, I. D. Mayergoyz, and G. Bertotti, J. Appl. Phys. 89, 6991 (2001).

34) S. Rohart and A. Thiaville, Phys. Rev. B 88, 184422 (2013).

35) A. A. Thiele, Phys. Rev. Lett. 30, 230 (1973).

36) J. Sampaio, V. Cros, S. Rohart, A. Thiaville, and A. Fert, Nat. Nanotechnol. 8, 839 (2013). 